\title{
A systematic review of the prevalence of sedentary behavior during the after-school period among children aged 5-18 years
}

\author{
Lauren Arundell", Elly Fletcher, Jo Salmon, Jenny Veitch and Trina Hinkley
}

\begin{abstract}
Background: Independent of physical activity levels, youth sedentary behaviors (SB) have negative health outcomes. SB prevalence estimates during discretionary periods of the day (e.g., after-school), inform the need for targeted period-specific interventions. This systematic review aimed to determine children's and adolescents' SB prevalence during the after-school period.

Methods: A computerized search was conducted in October 2015 (analysed November 2015). Inclusion criteria were: published in a peer-reviewed English journal; participants aged 5-18 years; measured overall after-school sedentary time (ST) objectively, and/or specific after-school SBs (e.g., TV viewing) objectively or subjectively; and provided the percentage of the after-school period spent in ST/SB or duration of behavior and period to calculate this. Where possible, findings were analyzed by location (e.g., after-school care/"other' locations). The PRISMA guidelines were followed.
\end{abstract}

Results: Twenty-nine studies were included: 24 included children ( $\leq 12$ years), four assessed adolescents ( $>12$ years) and one included both; 20 assessed ST and nine assessed SB. On average, children spent $41 \%$ and $51 \%$ of the after-school period in ST when at after-school care and other locations respectively. Adolescents spent $57 \%$ of the after-school period in ST. SBs that children and adolescents perform include: TV viewing (20\% of the period), non-screen based SB (including homework; $20 \%$ ), screen-based SB (including TV viewing; $18 \%$ ), homework/academics (13\%), motorised transport (12\%), social SB (9\%), and screen-based SB (excluding TV viewing; $6 \%$ ).

Conclusion: Children spent up to half of the after-school period in ST and this is higher among adolescents. A variety of screen- and non-screen based SBs are performed after school, providing key targets for interventions.

Trial registration: PROSPERO registration number CRD42015010437

Keywords: Children, Adolescents, Sedentary behavior, After-school hours, Prevalence

Abbreviations: METS, Metabolic equivalents; Cpm, Counts per minute; BEACHES, Behaviours of eating and activity for child health; SOFIT, System for observing fitness instruction time

\section{Background}

Sedentary behaviors are typically performed in a sitting/lying position and result in minimal energy expenditure ( $\leq 1.5$ metabolic equivalents [METS]) [1]. Evidence highlighting the negative health outcomes of sedentary behavior during childhood independent of physical activity levels is mounting [2]. For example, extensive TV viewing is positively associated with

\footnotetext{
* Correspondence: lauren.arundell@deakin.edu.au

Institute for Physical Activity and Nutrition Research, Deakin University, 221 Burwood Highway, Burwood, VIC, Australia
}

body composition and decreased academic achievement among children [2]. Many developed countries have endorsed recommendations that either place a limit on the time children should spend engaged in specific sedentary behaviors (e.g., Australia and Canada recommend less than $2 \mathrm{~h}$ of screen time per day $[3,4])$ or recommend minimising time spent sedentary (e.g., UK guidelines recommend minimising the time spent sedentary for extended periods $[5,6]$ ). Despite these guidelines, the majority of children exceed sedentary behavior recommendations [7-10]. 
One period of the day that has the potential to make a substantial contribution to children's daily sedentary behavior levels is the after-school period. During this period, children may have more choice over the behaviors they perform compared to other times of the day, such as during school hours. Further, children perform the majority of their recreational sedentary behavior during this period [11]. For example, children perform $84 \%$ of their daily screen-based sedentary behaviors and accrue $80 \%$ of the daily sedentary behavior guidelines (no more than two hours per day in front of screens) during the after-school period [11]. Although defined as 'the end-of-school to $6 \mathrm{pm}$ ' [12], many studies use a variety of definitions of after-school [13-15] which makes comparing the raw minutes engaged in sedentary behavior after school difficult as longer periods provide a greater opportunity to be sedentary. Therefore, identifying the percentage of time that children and adolescents engage in sedentary behavior after school is important for informing whether this period represents a potential intervention target.

Given public health guidelines focus on limiting screen-based sedentary behaviors as well as total sedentary time, both the prevalence of engagement in specific behaviors such as TV viewing and screen-time, as well as the total time spent sedentary during this period should be examined. This literature is yet to be synthesized and reviewed systematically. Therefore, the aim of this paper was to systematically review the percentage of time children and adolescents spend during the afterschool period in 1) 'sedentary time' defined as overall accumulated sedentary behavior measured objectively, and 2) distinct 'sedentary behaviors' particularly those pertinent to the guidelines (e.g., TV viewing), measured objectively or subjectively

\section{Methods}

This review is registered with PROSPERO (registration number: CRD42015010437).

\section{Search procedure}

A computerized search using the EBSCOhost search engine was conducted for peer-reviewed original research journal articles published in English before October 2015. The following databases were searched: Academic Search Complete, CINAHL Complete, Education Research Complete, MEDLINE, MEDLINE Complete, PsycARTICLES, Psychology and Behavioral Sciences Collection, PsycINFO and SPORTDiscus with Full Text. The key words in the search were age ("school age" OR youth OR young OR child* OR adolescen*), AND sedentary behavior (sedentar* OR television OR TV OR screen OR "electronic games" OR inactiv*), AND after-school period (after-school OR "after school” OR afternoon OR evening OR "critical window" OR "critical hours"). Reference lists of retrieved articles were also examined for potential papers. See Additional file 1: Table S1 for an example search strategy.

\section{Inclusion criteria}

Studies were eligible for inclusion if they incorporated children aged 5-18 years and used an objective measure to assess overall after-school 'sedentary time' and/ or used an objective or subjective measure to assess one or more individual after-school 'sedentary behaviors' (e.g., TV viewing, computer use). In addition, because of study variability in the duration of the afterschool period, the percentage of the after-school period spent in sedentary behavior or enough information for this to be calculated also needed to be reported. That is, the paper needed to report both the duration of sedentary behavior and the length of the after-school period whereby the proportion of the after-school period spent sedentary could be calculated as follows: (duration of sedentary behavior/ length of after-school period)*100. Studies were included if they examined behaviors in the afternoon once school had finished, regardless of the period start and finish times.

\section{Exclusion criteria}

Studies examining 'outside of school' sedentary behavior were excluded as this often included behaviors performed before school or on weekends. Studies of special populations (e.g., overweight/obese participants or children with a disability) were excluded to allow for generalizability to the broader population. Papers examining subjective measures of overall sedentary time (i.e., the total time children were sedentary) were excluded due to the variability in survey items and sedentary behaviors examined between studies (for example, studies included a variety of combinations of TV viewing, computer use, DVD use, homework etc.). This variance in the combination of individual behaviors as contributors to overall sedentary time would have prevented accurate comparisons with the objective measures of sedentary time or with other studies using a different combination of subjectively measured sedentary behaviors to constitute overall sedentary time.

Eligibility was initially determined through a review of the title and abstract by two authors (LA and EF, inter-rater reliability of initial screening was determined by percent agreement and found to be $83 \%$ agreement). The full-text of eligible studies were then located and reviewed. 


\section{Data extraction and synthesis}

Data for children (sample aged $\leq 12$ years) and adolescents (sample aged $>12-18$ years) were analyzed separately. Results for boys and girls are reported separately in this review if there were significant differences. All sedentary behaviors (e.g., TV viewing, motorised transport etc.) were examined to enable an exploration of the time spent in each behavior during the after-school period. The average proportion of the after-school period spent in sedentary time and each sedentary behavior was then calculated. Where accelerometry was used to assess overall sedentary time, results were separated and examined according to the accelerometer cut point used to provide a cut point-specific estimate of sedentary time. This was calculated by summing the proportion of the after-school period spent sedentary from all studies that used the cut point and dividing this by the number of those studies.

As the durations of the 'after-school' period varied greatly between studies (see Additional file 2: Table S2), comparisons were made via $\mathrm{t}$-tests of the estimated percentage of sedentary time among studies using an afterschool period of $\leq 180 \mathrm{~min}$ and $>180 \mathrm{~min}$ (based on the definition of end-of-school bell time to $6 \mathrm{pm}$ being approximately $180 \mathrm{~min}$ ). No differences were observed $(p=0.143)$, therefore all studies were analysed together. This also aligns with previous findings that compared the percentage of time spent sedentary during three after-school period lengths (end-of-school to $6 \mathrm{pm}$, endof-school to sunset and end-of-school to dinner time) and found no differences [12].

The PRISMA guidelines [16] were followed in reporting this review with the exception of conducting a methodological quality or risk of bias assessment. No methodological quality or risk of bias assessment was performed as the existing tools identified $[17,18]$ contained components specific to intervention or longitudinal studies. Therefore, those tools are not appropriate for prevalence reviews as previously noted in a systematic review of the prevalence of young children's ( $<2$ years) sedentary behavior [19]. Similarly, a previous systematic review of children's sedentary behavior prevalence [20] did not include assessment of risk of bias.

\section{Results}

Five-hundred and seventy papers were identified, screened and assessed for their eligibility (Fig. 1) and 29 studies met the inclusion criteria. Of these, 20 assessed overall after-school sedentary time and nine assessed after-school sedentary behaviors. Among the studies assessing overall sedentary time, 16 included children (sample aged $\leq 12$ years) [11-13, 21-33], three included adolescents (sample $>12-18$ years) [34-36], and one included both age groups [37]. Among the nine studies

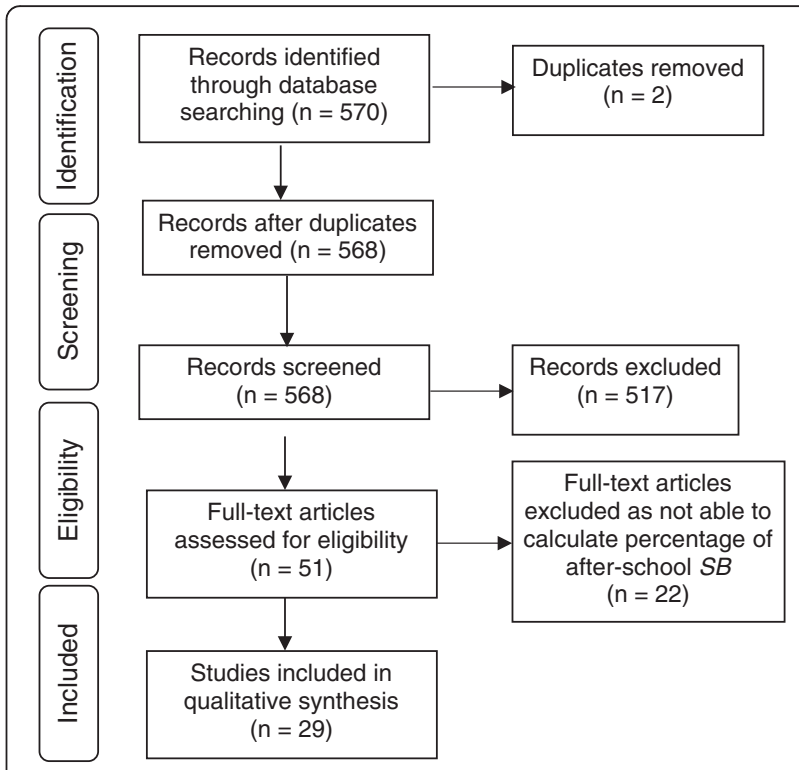

Fig. 1 Flow chart of results from systematic search conducted in 2015

assessing sedentary behaviors, eight included children [38-45], and one included adolescents [46]. The eligible papers were published between 1996 and October 2015 and were analysed in November 2015. Study samples ranged from 20 to 2053 (mean 578) and over half of the studies $(n=15)$ had a sample of fewer than 500 participants. Study characteristics can be found in Additional file 2: Table S2.

\section{Country of study}

Thirteen of the included studies were from the United States [22-24, 27, 29, 33, 35, 38, 39, 41, 42, 44, 45], with Australia [11, 12, 25, 43], Canada [30, 31] and the United Kingdom [13, 21, 28, 32, 37, 46] also having multiple studies. One study was identified from each of New Zealand [26], Ireland [34] and Portugal [36] and one study had a combined sample from Bulgaria, Taiwan and the United States [40].

\section{Child's after school location}

Among the 20 studies assessing after-school sedentary time, three assessed behavior while the children were at after-school care [22, 23, 29]. No studies examined adolescents' behaviors while at after-school care. The remaining 17 studies either did not report where the children were after school or noted that they were at a variety of places. These studies were grouped together as at 'other locations' [11-13, 21, 24-28, 30-37]. No studies investigating sedentary behaviors assessed behaviors when children were at after-school care, therefore all were considered 'other locations' $(n=9)$ [26, 38-46]. 


\section{After-school sedentary time Measurement tools}

The majority of studies measured sedentary time using the ActiGraph accelerometer $(n=15)$; however, a variety of cut points were used to indicate sedentary time including $<50$ counts per minute (cpm) [36], <100 cpm [11-13, 23, 26, $28,29,32,33,37],<300 \mathrm{cpm}[30,31],<800 \mathrm{cpm}$ [35], and $<1.5 \mathrm{METS}$ [24]. Of the remaining five studies, one used the Actical accelerometer with a sedentary cut point of $<100$ cpm [25], two used direct observation (modified version of BEACHES [Behaviours of Eating and Activity for Child Health] [27], and SOFIT [System for Observing Fitness Instruction Time] [22]), one used the RT3 tri-axial accelerometer with a cutpoint of $<288 \mathrm{cpm}$ [21] and one used the activPAL where sedentary was defined as sitting/lying down [34].

\section{Percentage of after-school sedentary time by sex}

Figure 2 shows the percentage of time children and adolescents spend sedentary during the after-school period by location, measure and cut point. On average, children spent $49.5 \%$ (range 16.1 - $88.9 \%$ ) and adolescents spent $56.6 \%$ (range 27.7 - $88.9 \%$ ) of the after-school period sedentary.

\section{Percentage of after-school sedentary time by location}

As shown in Fig. 2, results varied depending on the child's location. Children attending after-school care spent on average $41.1 \%$ (range 16.1-56.1 \%) [22, 23, 29, 33], and children at other locations spent on average $50.6 \%$ (range
$27.8 \%-73.5 \%)[11-13,21,24-28,30-33,37]$ of the afterschool period sedentary.

\section{Percentage of after-school sedentary time by cut point}

Results also varied when different cut points were used. Findings from accelerometer studies that examined sedentary time using $100 \mathrm{cpm}$ showed that children spent on average $42.3 \%$ of the after-school period sedentary $[11-13,23,25,26,28,29,32,33$, $37]$, whereas the average time children spent sedentary after school when using $<300 \mathrm{cpm}$ as the cut point $[30,31]$ was $71.2 \%$ of the period. Among adolescents, the studies that used $<50 \mathrm{cpm}$ [36] showed that on average adolescents spent $26.9 \%$ of the period sedentary and in comparison the studies that used $<800 \mathrm{cpm}$ [35] showed adolescents spent on average $82 \%$ of the after-school period sedentary.

\section{After-school sedentary behaviors Measurement tools}

A variety of subjective measurement tools were used to assess a range of after-school sedentary behaviors. Five studies assessed after-school TV viewing [38, 40, 43, 45, 46]. Seven studies reported screen-based sedentary behaviors and these were separated into two groups depending on whether or not their measure included TV viewing: four studies measured screen-based sedentary behaviors including TV viewing [39, 41, 42, 44] and three studies measured screen-based sedentary behaviors excluding TV viewing $[40,43,46]$. One study measured

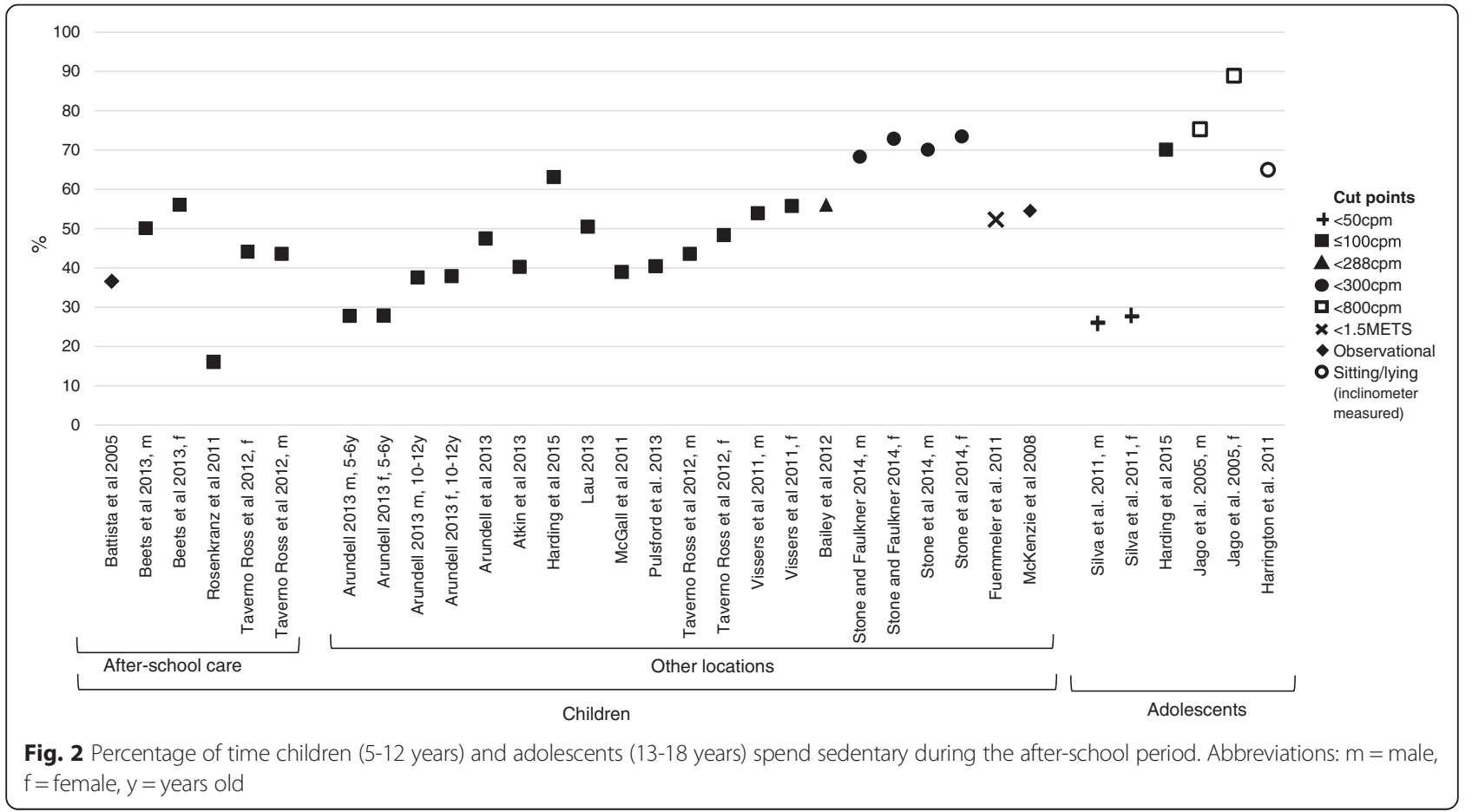


social sedentary behaviors [46], three measured homework/academics [43, 45, 46], three measured non-screen based sedentary behavior including homework/academics [41, 42, 44], one measured non-screen based sedentary behavior excuding homework/academics [43], and two measured motorised transport. [43, 46] The majority of studies used child self-report surveys asking children to report their after-school "free time" behaviors; [46] or previous day recall in 30-min blocks [41], one-hour blocks [40], in child-specific blocks (e.g., before/after child specified meal/snack) [43], or in $15 \mathrm{~min}$ intervals (via telephone interview) [42, 44, 45]. One study used parental proxy-report of behaviors in 15-min intervals [39] and another used observation to capture the time children spent watching TV [38].

\section{Percentage of time spent in specific after-school sedentary behaviors}

Figure 3 shows the percentage of the after-school period spent in specific sedentary behaviors. As only one study reported adolescents' after-school sedentary behaviors [46], these findings are presented alongside the children's after-school sedentary behavior studies [38-45]. Seven findings from five studies [38, 40, 43, 45, 46] reported the percentage of the after-school period spent watching TV. TV viewing averaged $20.4 \%$ (range 12.6 - $31 \%$ ) of the after-school period which was the highest percentage for any sedentary behavior (Fig. 3). The second largest percentage of the after-school period was spent performing non-screen based sedentary behaviors including homework (mean $20.3 \%$, range $10-29.2 \%$ ) [41, 42, 44]. This was followed by screen-based sedentary behaviors (including TV viewing;18.2 \%, range 8.5 - $25.3 \%$ ) [39, $41,42,44]$, homework/academics (12.9\%, range 6 $15.5 \%)[43,45,46]$, motorised transport (12.1\%, range 9.4 - $16.6 \%$ ) [43, 46], social sedentary behaviors (adolescent boys $7.9 \%$, girls $10.1 \%$ ) [46], screen-based sedentary behaviors (excluding TV viewing; $5.5 \%$, range 1.4 $8.3 \%)[40,43,46]$, and non-screen based sedentary behaviors excluding homework/academics, such as reading, sitting quietly, writing, playing cards/puzzles/board games $(3.7 \%)$ [43].

\section{Discussion}

This systematic review examined the prevalence of children's and adolescents' sedentary time and sedentary behaviors during the after-school period. The findings highlight that children spent between $41-51 \%$ of the after-school period sedentary and that adolescents are more sedentary than children (57 \%). TV viewing and other screen-based behaviors make up just $26 \%$ or less of this period. Other non-screen based sedentary behaviors (e.g., social sedentary behaviors, motorized transport, homework, and reading) comprise $54 \%$ of the after-school period; however, it is possible several of these behaviors occur concurrently [47, 48]. The percentage of time spent sedentary after school is greater than other periods of the day, such as recess and lunchtime, where children also have discretion over their behavior choices. For example, children aged 5-6 years and $10-12$ years spend approximately $15 \%$ and $14 \%$ of recess sedentary respectively, and $22 \%$ and $21 \%$ of lunchtime sedentary respectively [49]. Further, recess and lunchtime contribute $1.8 \%$ and $6 \%$ of children's daily

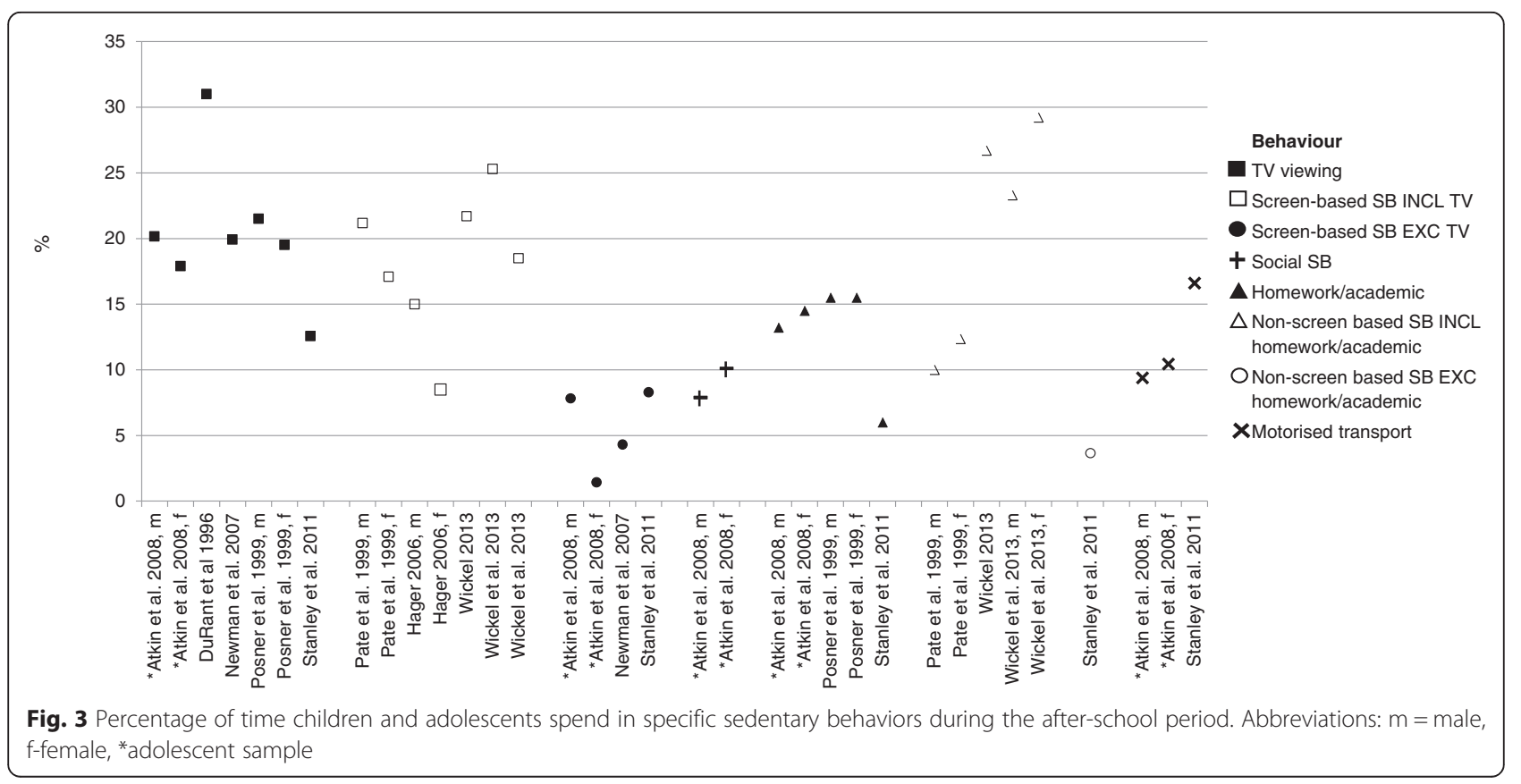


sedentary behavior time compared to the after-school period which contributes $26 \%$ of daily sedentary behaviors [21]. Subsequently, it would appear that the afterschool period is a key period that holds great potential for interventions to target reductions in sedentary time. Further, findings suggest that interventions may need to target other specific behaviors, such as motorised transport and social sedentary behaviors, in addition to screen-based sedentary behaviors which have traditionally been targeted for change.

This review also suggests that children spent less time sedentary when in after-school care compared with 'other locations'. This may be due to fewer sedentary pastime options (such as TV viewing and computer use) being available at after-school care. Further, after-school care may have more active structured and unstructured pastime options and facilities (e.g., active free play and organised sporting activities that use the school oval and sports equipment), and children may have more friends to be active with at after-school care compared to other locations. However, further research is needed to support this suggestion.

Findings from the included studies were highly variable. Children at after-school care are sedentary for $16.1 \%$ to $56.1 \%$ of the after-school period, children at other locations are sedentary for $27.8 \%$ to $73.5 \%$ of the period and adolescents are sedentary for $27.7 \%$ to $88.9 \%$ of the period. The substantial variability in estimates may be due to differences in sample sizes and accelerometer cut points. Although cut points $<100 \mathrm{cpm}$ may be capturing standing and light-intensity physical activity and incorrectly categorizing this as sedentary time, this threshold has been shown to most accurately represent sitting time when compared to inclinometers among 8-12 year-olds [50]. Based on findings using the cut point of $100 \mathrm{cpm}$, children spent approximately $25 \mathrm{~min}$ per hour of the after-school period sedentary and adolescents spent $42 \mathrm{~min}$ per hour of the afterschool period sedentary (although this was obtained from only one study among adolescents). However, a higher sedentary cut point can greatly elevate prevalence rates. For instance, Reilly and colleagues [51] found a $321 \mathrm{~min}(5 \mathrm{~h}, 21 \mathrm{~min})$ per day difference in sedentary time depending on the cut point used. This is also evident in the current review, as the prevalence of adolescents' after-school sedentary time ranged from $27 \%$ when using $<50 \mathrm{cpm}$ to $82 \%$ when using $<800 \mathrm{cpm}$. It is also important to note that there were large variations in estimates within thresholds. For example among the studies using $100 \mathrm{cpm}$, the percentage of time spent sedentary ranged from $16.1 \%$ [29] to $56.1 \%$ [32], highlighting the variability within the literature. This also highlights that there may be other important contextual factors impacting after-school behavior such as location (e.g., at home or after-school care) and who the children are with (e.g., alone or with friends) which require further investigation. Future research should also examine the intrapersonal, social and physical environment correlates which may further explain the variance in afterschool sedentary behaviors. Such investigation would enable identification of the characteristics of children and adolescents who display high levels of sedentary time and behaviors which can subsequently be used as intervention targets. Other factors, such as sample size and characteristics, may also explain part of the variance in prevalence rates.

The most frequently measured after-school sedentary behavior was TV viewing, with children and adolescents spending approximately one fifth of the after-school period watching TV. The percentage of the after-school period spent watching TV by children and adolescents was similar (children: $21 \%$ and adolescents $19 \%$ ) suggesting the age-related increases in after-school sedentary time observed in this review and previously observed [11] may be due to increases in participation in other sedentary behaviors (e.g., computer use or homework) [52]. However, it is hard to draw conclusions as only one study examined adolescents' sedentary behaviors. Further, the prevalence of after-school screen-based sedentary behaviors was more than three times higher when it included TV viewing compared to when the screen-based sedentary behavior measure did not include TV. This suggests that TV viewing is the main screen-based sedentary behavior after school and a potentially important intervention target if targeting screen-based sedentary behaviors.

The prevalence of screen-based sedentary behaviors that included TV viewing was lower than the prevalence reported by studies that just reported TV viewing. This may be due to the differences between measures used as differences in recall period and question response format (e.g., behaviors during previous day, previous week etc.) and the mode of administration (e.g., phone interview or written survey) may impact on the sedentary behaviors reported [53]. The studies examining screen-based sedentary behavior including TV viewing requested participants to report their present or previous day behaviors $[39,41,42,44]$ and asked for the main behavior being performed which does not allow for reporting of concurrent sedentary behaviors. In contrast, the studies examining TV viewing used a variety of recall periods from the present day [45] to recall of behaviors on three days [40]. There may be differences in daily TV viewing behaviors that are not able to be captured via a one day recall. Alternatively, higher prevalence rates may be due to $\mathrm{TV}$ viewing alone being an easier behavior to recall or participants may think broadly about all screens when asked about TV viewing without realising, whereas they 
may be more discriminatory when asked about individual screen use. Further, participants may have been performing sedentary behaviors concurrently, however this was not assessed. Additional exploration using consistent measures would facilitate direct comparisons.

It is also important to note that approximately onefifth of the after-school period was spent in non-screen based sedentary behaviors. Although only measured in three studies, it is possible that most of the non-screen based sedentary behaviors were homework or academic pursuits as when the measure did not include these behaviors, the prevalence was much lower $(3.6 \%)$. The similar percentage of time spent watching TV and in non-screen based sedentary behaviors suggests there may be opportunities for interventions to target sedentary behaviors other than TV viewing (e.g., through standing homework tasks [54]).

\section{Limitations of the current literature}

The objectively-measured sedentary time findings should be interpreted with some caution as there were numerous cut points used to represent sedentary time which may influence estimations of sedentary time. Also, while few studies reported children's behaviours while at afterschool care, the majority of studies did not report where the children or adolescents were after school resulting in these studies being combined into 'other locations'. The 'other locations' could include for example, a child/adolescent's home, a friend's or relative's home, or the local neighbourhood and there may be important differences in sedentary time/sedentary behaviors when children and adolescents are at such locations. However, this cannot be determined from the data available. Additional studies taking into account the child's/adolescent's actual location after school are important for informing the development of interventions targeting those settings where children are most likely to be sedentary during the after-school period. The variability of subjective measures of sedentary behaviors also limited the ability to directly compare findings. The use of uniform survey items in future studies would assist in gaining a greater understanding of the sedentary behaviors that children and adolescents perform after school. The varying period lengths makes direct comparisons of raw minutes of sedentary behavior after school difficult. While the use of proportion of time overcomes this, the use of the standardised definition of the after-school period (end-ofschool to $6 \mathrm{pm}$ [12]) would further facilitate the direct comparison of future studies examining after-school behaviors. As there were no studies that examined the individual sedentary behaviors children perform during after-school care and only one that examined the individual sedentary behaviors among adolescents, the evidence in these areas is limited. Sedentary behaviors performed during after-school care (e.g., seated crafts, board games) may differ to those at 'other' locations; therefore, this information may assist the development of interventions targeting the after-school care setting. Lastly, a limitation is that any bias due to study methodology quality within the current review is unknown as no methodological quality or risk of bias assessment was performed; therefore, higher quality studies may show a higher or lower prevalence rate than studies of poor quality. More appropriate measures of study quality for literature reviews of prevalence studies are needed to provide estimates based on the highest quality evidence.

\section{Conclusion}

Children and adolescents spent almost half of the afterschool period sedentary with adolescents spending a greater percentage of the period sedentary than children. Few studies measured behaviors performed while at after-school care; however, the limited evidence suggests that children spent less time sedentary at after-school care than at 'other' locations. Children and adolescents spent the greatest percentage of the after-school period watching TV and engaged in non-screen based sedentary behaviors; however, additional research is needed that measures other sedentary behaviors (e.g., mobile phone and digital tablet use, etc.), that uses standardized survey items to enable study comparisons, and that includes the adolescent population. This review highlights children's and adolescents' sedentary behaviors that can be targeted for reduction though interventions in the after-school period.

\section{Additional files}

Additional file 1: Table S1. Search strategy. Example of search strategy. (DOCX $15 \mathrm{~kb}$ )

Additional file 2: Table S2. Study characteristics. Details of the characteristics of the studies included in this review. (DOCX $23 \mathrm{~kb}$ )

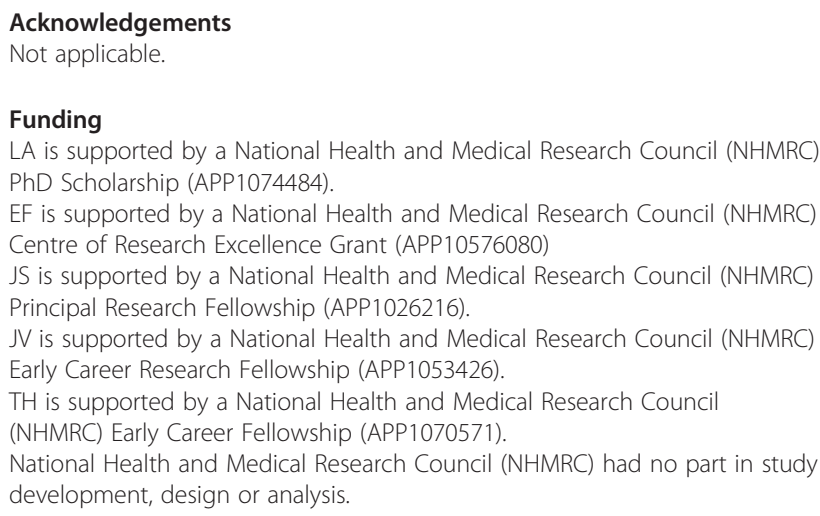

Availability of data and materials

The dataset supporting the conclusions of this article is included within the article (and its Additional files 1 and 2). 


\section{Authors' contribution}

$L A, E F$, JS, JV and TH were involved in the conception and design of the paper. LA and EF were involved in the data collection and synthesis. All authors contributed to the writing of the paper, read and approved the final manuscript.

\section{Competing interests}

The authors declare that they have no competing interests.

\section{Consent for publication}

Not applicable.

\section{Ethics approval and consent to participate}

Not applicable.

\section{Received: 2 March 2016 Accepted: 3 August 2016}

Published online: 22 August 2016

\section{References}

1. Sedentary Behaviour Research N. Letter to the editor: standardized use of the terms "sedentary" and "sedentary behaviours". Appl Physiol Nutr Metab. 2012:37(3):540-2. doi:10.1139/h2012-024.

2. Tremblay $M$, et al. Systematic review of sedentary behaviour and health indicators in school-aged children and youth. Int J Behav Nutr Phys Act. 2011;8(1):98. doi:10.1186/1479-5868-8-98.

3. Australian Government Department of Health. Australia's Physical Activity \& Sedentary Behaviour Guidelines for Children (5-12 years) 2014. Available from: http://www.health.gov.au/internet/main/publishing.nsf/content/ health-pubhlth-strateg-phys-act-guidelines\#apa512. Accessed 11 Nov 2015.

4. Tremblay $\mathrm{M}$, et al. Canadian sedentary behaviour guidelines for children and youth. Appl Physiol Nutr Metab. 2011;36(1):59-64.

5. Department of Health. Physical Activity Guidelines for Children and Young People (5-18 years). 2011

6. Department of Health (Scotland). Physical activity guidelines for children and young people (5-18 years). Scotland: Department of Health; 2011.

7. The NHS Information Centre. Statistics on obesity, physical activity and diet: England, February 2009. England: The NHS Information Centre for health and social care Part of the Government Statistical Service; 2009.

8. Troiano RP, et al. Physical activity in the United States measured by accelerometer. Med Sci Sports Exerc. 2008;40(1):181-8. doi:10.1249/mss. 0b013e31815a51b3.

9. Active Healthy Kids Canada. Healthy Habits Start Earlier Than You Think. In: The Active Healthy Kids Canada Report Card on Physical Activity for Children and Youth. Toronto: Active Healthy Kids Canada; 2010.

10. Australian Bureau of Statistics. Australian Health Survey: Physical Activity, 2011-12. cat no. 4364.0.55.004. Canberra: Commonwealth of Australia; 2013.

11. Arundell $L$, et al. 5-year changes in afterschool physical activity and sedentary behavior. Am J Prev Med. 2013;44(6):605-11. doi:10.1016/j. amepre.2013.01.029.

12. Arundell $L$, et al. Standardising the 'after-school' period for children's physical activity and sedentary behaviour. Health Promot J Austr. 2013;24(1): 65-7. doi:10.1071/HE12910.

13. Atkin AJ, et al. Determinants of change in children's sedentary time. PLoS One. 2013;8(6):1-9. doi:10.1371/journal.pone.0067627.

14. Pratt $C$, et al. Sedentary activity and body composition of middle school girls: the trial of activity for adolescent girls. Res Q Exerc Sport. 2008;79(4):458-67.

15. Biddle SJH, et al. Temporal and environmental patterns of sedentary and active behaviors during adolescents' leisure time. Int J Behav Med. 2009; 16(3):278-86. doi:10.1007/s12529-008-9028-y.

16. Cain KL, et al. Physical activity in youth dance classes. Pediatrics. 2015;135(6): 1066-73. doi:10.1542/peds.2014-2415.

17. National Collaborating Centre for Methods and Tools. Quality Assessment Tool for Quantitative Studies. Hamilton: McMaster University; 2008. Updated 13 April, 2010

18. Tooth $L$, et al. Quality of reporting of observational longitudinal research. Am J Epidemiol. 2005;161(3):280-8. doi:10.1093/aje/kwi042.

19. Downing $\mathrm{KL}$, et al. Prevalence of sedentary behavior in children under 2 years: A systematic review. Prev Med. 2015:105. doi:10.1016/j.ypmed.2015.07.019.

20. Hnatiuk JA, et al. A review of preschool children's physical activity and sedentary time using objective measures. Am J Prev Med. 2014:47(4):487-97. doi:10.1016/j.amepre.2014.05.042.
21. Bailey $D$, et al. Accelerometry-assessed sedentary behaviour and physical activity levels during the segmented school day in 10-14-year-old children: the HAPPY study. Eur J Pediatr. 2012;171(12):1805-13. doi:10.1007/s00431012-1827-0.

22. Battista J, et al. Elementary after school programs: an opportunity to promote physical activity for children. Calif J Health Promot. 2005;3(4):108-18.

23. Beets MW, et al. Impact of policy environment characteristics on physical activity and sedentary behaviors of children attending afterschool programs. Health Educ Behav. 2013:40(3):296-304. doi:10.1177/1090198112459051.

24. Fuemmeler BF, et al. Parent-child relationship of directly measured physical activity. Int J Behav Nutr Phys Act. 2011;8(1):17-25. doi:10.1186/1479-5868-8-17.

25. Lau J, et al. Parents' perceptions of children's physical activity compared on two electronic diaries. Pediatr Exerc Sci. 2013:25(1):124-37.

26. McGall SE. Contribution of free play towards physical activity guidelines for New Zealand primary school children aged 7-9 years. Br J Sports Med. 2011;45(2):120-4.

27. McKenzie TL, et al. Environmental correlates of physical activity in mexican american children at home. J Phys Act Health. 2008;5(4):579-91.

28. Pulsford RM, et al. Socioeconomic position and childhood sedentary time: evidence from the PEACH project. Int I Behav Nutr Phys Act. 2013;10(1): 105-13.

29. Rosenkranz RR, et al. Environmental correlates of objectively measured physical activity and sedentary behavior in after-school recreation sessions. J Phys Act Health. 2011;8:S214-S21.

30. Stone MR, Faulkner GEJ. Outdoor play in children: associations with objectively-measured physical activity, sedentary behavior and weight status. Prev Med. 2014;65:122-7. doi:10.1016/j.ypmed.2014.05.008.

31. Stone MR, et al. The freedom to explore: examining the influence of independent mobility on weekday, weekend and after-school physical activity behaviour in children living in urban and inner-suburban neighbourhoods of varying socioeconomic status. Int J Behav Nutr Phys Act. 2014:11(1):1-20. doi:10.1186/1479-5868-11-5.

32. Vissers PAJ, et al. Breakfast consumption and daily physical activity in 9-10year-old British children. Public Health Nutr. 2013;16(7):1281-90. doi:10.1017/ S1368980011002175

33. Taverno Ross SE, et al. After-school setting, physical activity, and sedentary behavior in 5th grade boys and girls. Health Place. 2012;18(5):951-5. doi:10. 1016/.healthplace.2012.06.013

34. Harrington DM, et al. Cross-sectional analysis of levels and patterns of objectively measured sedentary time in adolescent females. Int I Behav Nutr Phys Act. 2011; 8. doi:10.1186/1479-5868-8-120

35. Jago $R$, et al. Adolescent patterns of physical activity: differences by gender, day, and time of day. Am J Prev Med. 2005;28(5):447-52. doi:10.1016/j. amepre.2005.02.007.

36. Silva $\mathrm{P}$, et al. Seasonal differences in physical activity and sedentary patterns: The relevance of the PA context. J Sports Sci Med. 2011;10(1):66-72.

37. Harding SK, et al. Longitudinal changes in sedentary time and physical activity during adolescence. Int J Behav Nutr Phys Act. 2015; 12 . doi:10. 1186/s12966-015-0204-6

38. DuRant $\mathrm{RH}$, et al. The relationship among television watching, physical activity, and body composition of 5- or 6-year-old children. / Relation entre le temps passe devant la television, l'activite physique et la composition metabolique corporelle chez des enfants ages de 5 ou 6 ans. Pediatr Exerc Sci. 1996:8(1):15-26

39. Hager RL. Television viewing and physical activity in children. J Adolesc Health. 2006;39(5):656-61.

40. Newman J, et al. What do they usually do after school? J Early Adolesc 2007:27(4):431-56.

41. Pate RR, et al. Tracking of physical activity, physical inactivity, and healthrelated physical fitness in rural youth. Pediatr Exerc Sci. 1999:11(4):364-76.

42. Wickel E. Variables associated with active and inactive behavior during the after-school period. Pediatr Exerc Sci. 2013:25(2):288-99.

43. Stanley RM, et al. The type and prevalence of activities performed by Australian children during the lunchtime and after school periods. J Sci Med Sport. 2011;14(3):227-32.

44. Wickel EE, et al. Longitudinal change in active and sedentary behavior during the after-school hours. J Phys Act Health. 2013;10(3):416-22.

45. Posner JK, Vandell DL. After-school activities and the development of lowincome urban children: a longitudinal study. Dev Psychol. 1999;35(3):868-79.

46. Atkin AJ, et al. Critical hours: physical activity and sedentary behavior of adolescents after school. Pediatr Exerc Sci. 2008;20(4):446-56. 
47. Gorely T, et al. Couch kids: correlates of television viewing among youth. Int J Behav Med. 2004;11:152-63. doi:10.1207/s15327558ijbm1103_4.

48. Marshall SJ, et al. Clustering of sedentary behaviours and physical activity among youth: a cross-national study. Pediatr Exerc Sci. 2002;14:401-17.

49. Ridgers ND, et al. Five-year changes in school recess and lunchtime and the contribution to children's daily physical activity. Br J Sports Med. 2011. doi: 10.1136/bjsm.2011.084921.

50. Ridgers ND, et al. Agreement between activPAL and ActiGraph for assessing children's sedentary time. Int J Behav Nutr Phys Act. 2012;9:15. doi:10.1186/ 1479-5868-9-15.

51. Reilly J, et al. Objective measurement of physical activity and sedentary behaviour: review with new data. Arch Dis Child. 2008;93:614-9. doi:10. 1136/adc.2007.133272.

52. Veitch J, et al. Is the Neighbourhood Environment Associated with Sedentary Behaviour Outside of School Hours among Children? Ann Behav Med. 2011. doi:l 10.1007/s12160-011-9260-6.

53. Lau EY, et al. Associations between home environment and after-school physical activity and sedentary time among 6 th grade children. Pediatr Exerc Sci. 2015;27(2):226-33. doi:10.1123/pes.2014-0061.

54. Salmon J, et al. A cluster-randomized controlled trial to reduce sedentary behavior and promote physical activity and health of 8-9year olds: the TransformUs! study. BMC Public Health. 2011;11:759. doi:10.1186/1471-2458-11-759.

Submit your next manuscript to BioMed Central and we will help you at every step:

- We accept pre-submission inquiries

- Our selector tool helps you to find the most relevant journal

- We provide round the clock customer support

- Convenient online submission

- Thorough peer review

- Inclusion in PubMed and all major indexing services

- Maximum visibility for your research

Submit your manuscript at www.biomedcentral.com/submit
Biomed Central 